\title{
Informes
}

\section{PROGRAMA DE POSGRADO EN POBLACIÓN DEL CENTRO DE ESTUDIOS DEMOGRÁFICOS Y DE DESARROLLO URBANO (CEDDU) DE EL COLEGIO DE MÉXICO}

Doctorado en ciencias sociales con especialidad en estudios de población, 1985

La experiencia de veinte años en la investigación y formación de recursos en población, y la voluntad de continuar respondiendo a las necesidades y requerimientos del país y de la región en este campo, llevan al CEDDU de El Colegio de México en 1985 a una etapa que se concreta en un programa de Doctorado en Ciencias Sociales con especialidad en Estudios de Población. Este doctorado forma parte del Doctorado en Ciencias Sociales que ofrece El Colegio de México.

El objetivo del programa tiene como eje fundamental formar investigadores, ofreciendo el espacio para una reflexión más profunda en la manera de analizar la integración de los procesos demográficos y de cambio social. Esta reflexión se plantea a partir de una formación básica en las herramientas del análisis demográfico, articulándola con el manejo amplio de los avances teóricos en los estudios de población, principalmente dentro de la sociología y la economía.

El programa de estudios de doctorado tiene una duración mínima de dos años, a partir de nivel de maestría, durante los cuales el estudiante, a través de seminarios, debe cubrir áreas teórico-metodológicas y de especialización y avanzar sustancialmente en la investigación de un tema específico de la relación población-desarrollo. La realización de este programa se apoya en un cuerpo permanente de profesores investigadores del CEDDU, y se enriquece con aportes académicos de otros centros de estudios de El Colegio de México.

Para completar el programa y obtener el grado de doctorado el estudiante debe: haber aprobado los seminarios y cursos, y someter y defender satisfactoriamente una tesis doctoral, que demuestre su capacidad como investigador y donde haga una aportación original en el campo. 
Maestría en demografía 1985-1987

En septiembre de 1985 inició su programa de labores la doceava promoción de la maestría en demografía del CEDDU. Desde su inicio en 1964, el Programa de Maestría en Demografía tiene como objetivo principal el de formar especialistas en el análisis de los fenómenos demográficos y sus interrelaciones con el proceso de desarrollo y cambio social, con especial énfasis en la problemática de México y de América Latina. Con la orientación interdiscipl inaria privilegiada desde los inicios del programa, la maestría busca proporcionar una base sólida en el instrumental propio del análisis demográfico, ofreciendo simul táneamente en los seminarios de población una revisión sistemática y crítica de los enfoques teóricos y de los resultados de los principales estudios en fecundidad, mortalidad, migración y fuerza de trabajo. Asimismo, se ofrecen cursos de economía, sociología y de aspectos instrumentales de matemáticas y estadística.

En los últimos dos semestres se introduce al estudiante en la práctica de investigación propiamente dicha, por medio de cursos de metodología y seminarios de tesis, así como de seminarios optativos que le permitan profundizar en las áreas de su elección. Entre estos últimos se pueden mencionar los seminarios sobre fuerza de trabajo y migraciones, dinámica poblacional y estructura agraria, y demografía matemática.

\section{CONGRESO GENERAL DE LA UNIÓN INTERNACIONAL PARA EL ESTUDIO CIENTÍFICO DE LA POBLACIÓN}

La Unión Internacional para el Estudio Científico de la Población es una de las más prestigiadas asociaciones científicas a nivel mundial, con más de 2000 miembros.

Cada cuatro años se realiza la reunión general donde se presentan los resultados de las líneas de investigación que han sido prioritarias en los últimos años. En ella se discuten aspectos teóricos y metodológicos, en ocasiones polémicos, y se perfilan las líneas futuras de investigación.

En esta ocasión el XX Congreso General se llevó a cabo del 5 al 12 de junio de 1985 en Florencia, Italia. Modificando los reglamentos seguidos en el pasado, en las sesiones formales se incluyeron documentos espontáneos seleccionados por los organizadores de cada mesa, además de aquellos documentos solicitados, lo que permitió la participación de expertos en el tema y de investigadores más jóvenes.

El programa de la conferencia, en parte, es la culminación de actividades científicas que realizan los ocho comités y tres grupos de trabajo sobre las líneas de investigación prioritarias acordadas durante los cuatro años anteriores al Congreso. Los comités y grupos de trabajo cubren diversos aspectos de la problemática poblacional; éstos son: 
1) Comité sobre el análisis comparativo de la fecundidad y la planificación familiar;

2) Comité sobre la utilización del conocimiento demográfico en la formulación de políticas y planeación;

3) Comité sobre las consecuencias económicas debido a patrones de población alternativos;

4) Comité sobre las asociaciones biológicas y sociales de la mortalidad;

5) Comité sobre los aspectos económicos y sociales de la migración internacional;

6) Comité sobre la demografía de la familia y el ciclo de vida;

7) Comité sobre migración internacional;

8) Comité sobre demografía histórica;

9) Grupo de trabajo sobre recolección, evaluación y ajuste de datos demográficos en países menos desarrollados;

10) Grupo de trabajo sobre bibliografías nacionales;

11) Grupo de trabajo sobre enfoques de micro-demografía.

Con base en el trabajo realizado por los comités y grupos de trabajo y en las líneas prioritarias en los estudios de población, la conferencia englobó a éstas en ocho sesiones formales y dos plenarias (y 14 mesas redondas, sesiones informales).

Sería difícil resumir en unas cuantas líneas los trabajos presentados y las discusiones surgidas en esta conferencia; además creemos que una presentación de este tipo sería muy parcial. Hemos preferido presentar los temas que se abordaron en la conferencia, y finalizar con unos comentarios generales.

Sesiones formales

A. Fecundidad

F.1 Influencias contextuales, la sociedad y la comunidad en la fecundidad individual.

F.2 Nutrición, mortalidad infantil y fecundidad.

F.3 Aborto, esterilización y su relación con la fecundidad.

F.4 Implicaciones del descenso de la fecundidad en países en vías de desarrollo.

\section{B. Mortalidad}

F.5 Marcos de referencia conceptuales para el estudio de los determinantes sociobiológicos de la mortalidad.

F.6 Mortalidad en África.

F.7 Factores asociados con el descenso de la mortalidad en países de altos niveles de mortalidad. 
F.8 Patrones y tendencias en las causas de muerte en paises con baja mortalidad.

C. Desarrollo, migración y redistribución de la población

F.9 Consecuencias demo-económicas de la migración internacional.

F.10 Patrones de asentamiento y sus implicaciones demográficas.

F.11 Crecimiento poblacional urbano y sus consecuencias socioeconómicas.

F.12 Dinámica de la fuerza de trabajo y la migración internacional.

D. Demografía de la familia, ciclo de vida y la nupcialidad

F.13 Factores demográficos y otros en el ciclo de vida de la familia.

F.14 Cambios en la formación y disolución de familias en paises menos desarrollados.

E. Polfticas y el comportamiento individual

F.15 Utilización del conocimiento demográfico en la formulación de políticas y planeación.

F.16 Respuestas a políticas orientadas a aumentar la fecundidad en paises de baja fecundidad.

F.17 Planificación familiar: prospectos para el futuro.

F.18 Envejecimiento, seguridad social y comportamiento económico.

F. Metodologia

F.19 Nuevos métodos de ajuste y nuevos enfoques de encuestas para la estimación demográfica.

F.20 Problemas estadísticos de relevancia en la recolección de datos estadísticos en paises en vías de desarrollo.

F.21 Avances en proyecciones demográficas.

F.22 Demografía matemática.

F.23 Contribución de los micro-estudios al conocimiento demográfico.

G. Patrones de crecimiento de la población

F.24 Oscilaciones a largo plazo de la población en el pasado y sus determinantes.

F.25 Patrones presentes de cambios demográficos a la luz de experiencias en el pasado.

F.26 Las relaciones de cambio entre características socioeconómicas y el comportamiento demográfico. 
H. Tópicos especificos para áreas regionales

F.27 El censo de China, 1982.

F.28 La demografía de África a la luz de la ronda de los censos de 1980.

Sesión plenaria 1

Visión demográfica del siglo XXI.

Sesión plenaria 2

Tributo a Giorgio Mortara.

Políticos y Académicos: responsabilidades compartidas en los aspectos poblacionales.

En las sesiones plenarias surgieron dos puntos importantes: el giro que necesariamente deben dar los estudiosos sobre población, orientado más a sus explicaciones socioeconómicas, y la importancia que revisten los aspectos de salud.

Cabe señalar que aunque en muchas regiones la parte explicativa, referida a los aspectos socioeconómicos y políticos del tema poblacional, ha sido descuidada, en América Latina esta línea de investigación ha sido prioritaria con avances teórico-metodológicos importantes.

El estudio del proceso de salud y su vinculación a los estudios demográficos, aunado al levantamiento de una serie de encuestas sobre salud y demografía, marcan la apertura a estos interesantes aspectos menos estudiados a la fecha.

En general, las sesiones intentaban guardar un balance entre las posibles temáticas de los estudios poblacionales y al mismo tiempo dar representatividad a las áreas geográficas estudiadas. Sin embargo, es en este foro internacional donde surgen algunos cuestionamientos por parte de los miembros de los países menos desarrollados. Aunque los temas presentados analizan datos y problemáticas de los países desarrollados y de aquellos en vías de desarrollo, los ponentes, los participantes, los responsables de los comités científicos, así como los directivos de la Unión, provienen en su mayoría de países desarrollados.

Resulta obvio que es precisamente en los países menos desarrollados donde se requiere el mayor financiamiento para investigaciones locales y el impulso a la formación de especialistas; sin embargo, las tendencias económicas y políticas que separan a los países desarrollados y a los en vías de desarrollo no han podido romperse ni siquiera en el plano académico.

Fátima Juárez 
SEMINARIO INTERNACIONAL SOBRE

"LAS GRANDES METRÓPOLIS DEL MUNDO"

En los últimos tiempos el problema de las grandes ciudades ha atraído la atención de estudiosos y políticos. Numerosos eventos internacionales se han llevado a cabo para tratar los difíciles problemas de esos centros urbanos; muchos de ellos han reunido sobre todo a responsables de la administración de los mismos.

El seminario realizado en Barcelona, en febrero de 1985, organizado conjuntamente por el Consejo Internacional de Ciencias Sociales de la UNESCO y la Corporación Metropolitana de Barcelona, ha congregado fundamentalmente a investigadores en el área de estudios urbano-regionales, los que presentaron y discutieron 25 documentos referidos a:

1) Los patrones de urbanización en las grandes regiones del mundo (Europa occidental y oriental; Norteamérica y América Latina, África y el sureste de Asia).

2) Los problemas particulares de 14 grandes metrópolis, y

3) Algunos aspectos específicos de las grandes ciudades, incluyendo desde la degradación ambiental hasta los problemas de la restructuración económica.

Pero también, en un coloquio final con el cual culminó la reunión, se elaboraron recomendaciones y conclusiones, con la participación de políticos y responsables de la administración de algunas grandes ciudades. Creemos que justamente la combinación de los aportes de investigadores, políticos y administradores dio, a la Declaración de Barcelona, un interés que vale la pena revelar en esta presentación.

La Declaración comienza afirmando que el efecto combinado de la crisis económica (que ha agravado los problemas sociales) y del fracaso relativo de la planificación, han provocado una gran pérdida de confianza en la capacidad de la sociedad para reconstruir ciudades humanas y eficientes.

Luego, se hace referencia a los problemas de las metrópolis tanto en países subdesarrollados como desarrollados, partiendo de la premisa que la disminución del crecimiento de aquéllas no puede, por sí misma, resolver los problemas y los déficit. ${ }^{1}$

\footnotetext{
1 Muchas de las metrópolis presentadas en el seminario han mostrado un crecimiento explosivo, y tendrán para el año 2000 cifras de población elevadísimas. La ciudad de México, 30 millones de habitanteś; San Pablo, 26 millones; Lagos, 13 millones y Shangai, 22 millones.

Las grandes metrópolis de los países desarrollados crecen mucho menos, y en muchos casos la ciudad central pierde bastante población, pero aumentan los grupos de menores ingresos y los problemas de empleo.

Para el año 2000 se prevé que las metrópolis de más de 5 millones de habitantes crecerán en conjunto de 260 millones a 658 millones de habitantes, de los cuales $70 \%$ corresponderá a los países subdesarrollados.
} 
En las grandes metrópolis del "subdesarrollo" son cada día más graves los enormes déficit de vivienda e infraestructura urbana, la pobreza y la marginalidad de una parte importante de la población, la degradación del medio-ambiente y la insuficiencia y alto costo de los servicios públicos.

Esos problemas también se presentan, aunque en menor grado, en los países desarrollados. Sin embargo, con la crisis, la pérdida de base productiva, la marginalidad de un sector de la población, la violencia y la inseguridad parecen agravarse.

A pesar de los problemas señalados, se afirma en la Declaración que las grandes metrópolis presentan aspectos positivos; ${ }^{2}$ y que por lo tanto resulta necesario defender el patrimonio que ellas representan y reconocer las potencialidades que contienen, tanto a nivel nacional como mundial.

Se considera que la gran ciudad debería tomar la delantera en los procesos de reconversión económica y ser la protagonista del desarrollo de nuevas tećnologías, promoviendo el entrenamiento de los jóvenes y apoyando iniciativas para generar nuevos empleos. Así, los gobiernos de esos grandes centros deberian, por ejemplo, estimular las iniciativas económicas de empresas medianas y pequeñas. Si para las actividades orientadas a la exportación esos centros deben ser competitivos, para aquellas destinadas a la satisfacción de necesidades locales sería conveniente utilizar recursos propios para lograr la creación de empleos y la integración cultural.

Para cumplir los objetivos económicos, el gobierno local de las metrópolis deberia realizar reformas administrativas que sustituyeran las formas tradicionales de gestion, introduciendo programas orientados hacia objetivos precisos, basados en la autonomía de los órganos sectoriales y territoriales. Esto permitiría la creación de estructuras flexibles y competentes en las áreas de apoyo a la promoción de la innovación económica y la cooperación social.

Por otra parte, el documento manifiesta que el desarrollo urbano debería tratar de lograr la renovación y rehabilitación de las ciudades, a corto plazo, evitando el desperdicio de recursos y de espacio, y mejorando, al mismo tiempo, la comunicación y la intensidad de contactos. Debería luchar contra la segregación a la atomización, contra la pobreza y la marginalidad, así como contra la tendencia a la privatización de los espacios públicos.

A continuación se hace hincapié en la necesidad de apoyar la autonomía y representatividad de los gobiernos locales:

"La descentralización a favor de gobiernos locales es la mejor respuesta a la crisis política y financiera de la administración pública."

La conveniencia de aplicar una política metropolitana que promueva

2 Por ejemplo, infraestructura material y técnica, una población calificada y diversificada, concentración de recursos políticos y financieros, capacidad de investigación. creatividad e innovación, etcétera. 
el desarrollo económico, que logre una reordenación del territorio, que asegure los servicios públicos y que colabore en la redistribución del ingreso, requiere de la creación y el fortalecimiento de gobiernos metropolitanos, sin disminuir la importancia de los municipios que forman parte dé las áreas metropolitanas.

Siguiendo con el tema de la gestión urbana, se pone énfasis en el problema de la legitimidad democrática del gobierno de las grandes ciudades. Se propone el establecimiento de procedimientos electorales que aseguren la representatividad de todos los sectores sociales, y se afirma que la participación de los ciudadanos es una condición indispensable para la eficacia de la administración pública y para la democratización del gobierno.

La declaración termina destacando el papel dinámico e innovador que le corresponde a las organizaciones y movimientos de ciudadanos, que juegan un papel muy importante en la resolución de los problemas inmediatos de la ciudad, y que están en condiciones de proponer nuevas alternativas para el desarrollo social y la administración pública.

Martha Schteingart 